\title{
ABC Autismo - Uma Aplicação Mobile para Auxiliar no Processo Alfabetizador de Crianças com Autismo
}

\author{
Ezequiel B. Farias ${ }^{1}$, Mônica X. C. Cunha ${ }^{2}$, Jose W. S. Souza ${ }^{1}$ \\ ${ }^{1}$ Instituto da Computação - Universidade Federal de Alagoas (UFAL) - Maceió - AL - \\ Brasil \\ ${ }^{2}$ Coordenadoria de Informática - Instituto Federal de Alagoas (IFAL) - Maceió, AL - \\ Brasil \\ ebfeic.ufal.br, mxcc@hotmail.com, wellison.maceio@gmail.com
}

\begin{abstract}
Resumo: Dentro do campo educacional a tecnologia tem quebrado inúmeras barreiras e potencializado o processo de aquisição de conhecimento. Entretanto se a mesma não for apoiada em modelos de ensino eficazes e conceitos consolidados, seu uso pode trazer prejuízos para a vida de seus utilizadores, como exemplo disso temos a população autista. Por ser uma síndrome caracterizada por falhas em áreas específicas e em níveis diversos, a mesma acaba exigindo adequação dos mecanismos utilizados para o tratamento de indivíduos e tecnologias que não estejam alicerçadas em modelos efetivos de tratamento podem prejudicar o processo de aquisição de conhecimento. Sendo assim foi desenvolvido o ABC Autismo, aplicação móvel baseada na abordagem TEACCH, voltada para o processo de alfabetização de crianças com autismo.
\end{abstract}

\section{Cenário de Uso}

O autismo é uma condição neurobiológica que se manifesta antes dos três anos de idade e perdura durante toda vida, afetando basicamente três áreas no indivíduo: a comunicativa, a social e a comportamental (Felicio, 2007). Indivíduos com autismo possuem prejuízos qualitativos em suas aptidões coletivas e comunicativas, resultando em características como impulsividade, desatenção, crises de "birra". Além disso, o autista pode ter um acentuado défice no uso de vários comportamentos não verbais, tais como o contato ocular, expressão facial, postura corporal e gestos reguladores da interação social (Vila, 2009).

Todas estas peculiaridades oriundas do espectro autista acabam por dificultar o processo de aquisição do conhecimento, sendo em muitos casos inviável a transmissão do saber pelos moldes convencionais de ensino. Apesar de existirem inúmeras barreiras a serem superadas nesse processo, o que torna a aprendizagem de crianças autistas uma tarefa difícil, acredita-se que não seja impossível prover ensino de qualidade a crianças que vivem esta realidade (Cunha, Farias, 2013). Tal afirmação apoia-se em estudos que defendem a intervenção multidisciplinar como modelo de tratamento efetivo dentro da conduta autista (Orrú, 2009).

Varias abordagens voltadas para o desenvolvimento cognitivo deste público foram desenvolvidas nas últimas décadas, segundo Mello et al. (2013) as mais utilizadas dentro das associações e centros de apoio espalhados pelo nosso país são o TEACCH, o ABA e o PECS respectivamente. 
Uma abordagem que vem obtendo bons resultados com relação ao tratamento e ensino de pessoas com autismo é o TEACCH, um programa criado em 1964, na Universidade da Carolina do Norte (EUA), por Eric Schoppler e colaboradores (Farias et al., 2014). O programa TEACCH trabalha essencialmente com a estruturação do tempo, atividades, materiais e ambientes utilizados pela criança visando compensar os déficits característicos do autismo e proporcionar ganhos significativos para o convívio social.

É sabido que a maioria dos autistas não aprende naturalmente em ambientes normais, no entanto, sob orientações e instruções apropriadas, muitos deles podem aprender uma grande variedade de conteúdos (Felicio, 2007). Desta forma a abordagem TEACCH fornece um modelo a ser utilizado para adaptação de conteúdo ao público autista. Esta adaptação é direcionada não só ao processo de elaboração das atividades como também para o processo de execução.

Essa estrutura oriunda da abordagem torna possível o ensino de uma ampla gama de conceitos e habilidades a crianças com autismo, dentro deste contexto pode ser colocado o processo de alfabetização, fundamental para a formação intelectual de qualquer indivíduo e base para obtenção de outros conhecimentos necessários para a autonomia do indivíduo.

É importante frisar que todo este processo de adaptação realizado dentro da abordagem TEACCH acaba sendo oneroso para os profissionais que utilizam o modelo interventivo, visto que as tarefas adaptadas são elaboradas individualmente, de forma artesanal, o que de certa forma dificulta o trabalho de quem as elaboram, uma vez que precisam ser constantemente confeccionadas e variadas, a fim de acompanhar os níveis de evolução do tratamento da criança. Além disso, a repetitividade das tarefas elaboradas, por ser uma das premissas da abordagem TEACCH, além de propiciar uma aquisição mais efetiva das habilidades trabalhadas, fornece um caráter dinâmico a todo o processo.

Procurando superar este obstáculo o aplicativo $\mathrm{ABC}$ Autismo foi criado com o intuito de auxiliar no processo alfabetizador de crianças com autismo. O ABC Autismo incorpora alguns aspectos de uma abordagem manual, o TEACCH, método amplamente difundido e comprovadamente eficaz na conduta autista. $\mathrm{O}$ aplicativo em questão utiliza uma plataforma móvel, foi desenvolvido inicialmente para Tablets com o sistema operacional Android, e foca diretamente no ensino de habilidades necessárias para a alfabetização da criança autista, tais como habilidades de correspondência, pareamento e letramento inicial.

\section{Desenvolvimento}

Objetivando elaborar uma ferramenta alinhada ao programa TEACCH, que possa realmente beneficiar o tratamento das crianças com autismo, todo um processo foi realizado procurando obedecer às etapas necessárias para a construção de um aplicativo eficiente e eficaz.

Inicialmente se fez necessário compreender o universo autista, as características, variantes e demais peculiaridades, que servissem de base, posteriormente, para a implementação das principais funcionalidades do aplicativo. Com este intuito uma pesquisa exploratória, qualitativa, foi realizada. 


\section{CBIE-LACLO 2015}

Anais dos Workshops do IV Congresso Brasileiro de Informática na Educação (CBIE 2015)

Procurando validar todo o material colhido na pesquisa inicial, uma parceria foi realizada com a Associação de Amigos dos Autistas de Alagoas (AMA-AL), associação de caráter assistencial sem fins lucrativos que utiliza o Programa TEACCH como base em seus tratamentos. A AMA-AL, espaço que propiciou o estudo, é formada por pais de crianças com autismo, que buscam evolução no quadro clínico de seus filhos. Criada em 2008, a AMA-AL atende aproximadamente 30 crianças na faixa etária de 02 a 13 anos, com diagnóstico de transtorno invasivo do desenvolvimento e residentes em Maceió.

Visando explorar a estrutura oferecida pela AMA-AL às crianças, foram realizadas uma série visitas à sede da associação, onde foi possível acompanhar os processos interventivos que aplicavam a abordagem TEACCH. Acompanhada dessas visitas uma série de entrevistas foi realizada com o intuito de levantar os requisitos iniciais relacionados aos procedimentos utilizados na associação, especialmente aqueles relacionados ao programa TEACCH.

Cada aspecto relacionado à abordagem TEACCH foi cuidadosamente investigado, dentre estes podem ser citados: o entendimento de como o ambiente é estruturado para receber as crianças, como é realizado o processo de elaboração das atividades adaptadas e como as mesmas são distribuídas entre as crianças, quais são as áreas destinadas para armazenamento, execução e descarte utilizadas, como se dá a mediação dos profissionais durante o tratamento, quais os incentivos dados à criança durante o tratamento, quais os níveis de recompensas e dicas apresentados, dentre outros.

Todas as ações realizadas dentro da visita de campo foram registradas na forma de relatórios, que por sua vez deram suporte para a execução do processo de especificação do aplicativo com o desenvolvimento dos modelos de telas, diagramação dos elementos e documentação, visando explicitar todas as funcionalidades necessárias para compor a ferramenta neste primeiro momento.

Uma vez levantadas e validadas todas as características iniciais do aplicativo e ciente das peculiaridades oriundas do espectro autista, foi realizado um estudo de viabilidade com o intuito de definir qual a linguagem de programação a ser utilizada para o desenvolvimento da ferramenta. Neste estudo a linguagem Java, voltada para sistemas operacionais Android, acabou se sobressaindo em relação às outras linguagens investigadas. As razões que mais impactaram na escolha foram à portabilidade, pelo fato de ser uma linguagem totalmente aberta, que beneficiará qualquer tentativa de adaptação do aplicativo para outras plataformas, além da facilidade de manutenção devido à existência de inúmeras comunidades de desenvolvimento com foco na linguagem.

Após todo o processo de levantamento dos requisitos necessários para o funcionamento da ferramenta foram elaborados os diagramas de caso de uso, bem como toda a modelagem dos módulos iniciais. Neste processo, foram utilizadas ferramentas que auxiliaram no processo, como o Evolus Pencil ${ }^{1}$ para a elaboração dos modelos de tela e o Astah Comunnity ${ }^{2}$ para a diagramação do projeto. A criação dos modelos iniciais de telas foi suportada por uma pesquisa em sites da internet por imagens, desenhos que pudessem retratar visualmente, o mais fidedignamente possível, a estrutura física do ambiente proposto pela abordagem TEACCH. Parte das imagens

\footnotetext{
Evolus Pencil é uma ferramenta para design de interfaces.

${ }^{2}$ Astah Community é um software para modelagem UML.
} 
encontradas na internet foi utilizada para a elaboração de um banco de imagens usado durante toda a fase de prototipação representando os elementos utilizados dentro de cada tarefa elaborada.

Concluídas as etapas anteriores, uma serie de reuniões foram realizadas com o intuito de definir a metodologia de desenvolvimento a ser utilizada durante processo de implementação do aplicativo. A metodologia $\mathrm{Scrum}^{3}$ foi a que mais se adequou a especificidades e prazos do projeto. Sendo uma metodologia ágil de desenvolvimento, a mesma permitiu gerar pouca documentação durante o processo o que permitiu um melhor controle sobre o desenvolvimento. Cada milestone do processo de codificação foi dividido em Sprints ${ }^{4}$, estórias de usuários elaboradas em parceria com os profissionais entendedores do programa TEACCH base para o aplicativo. Cada uma das Sprints teve um prazo previamente estabelecido para entrega e foram minunciosamente revisadas permitindo melhorias constantes na implementação de funcionalidades para a ferramenta. O processo de codificação do aplicativo foi realizado em constante contato com profissionais entendedores da problemática autista, o que representou um forte diferencial para o desenvolvimento.

Uma vez concluídos os esforços relacionados à construção da ferramenta móvel, um processo de validação (última fase do desenvolvimento) foi realizado, através da execução do aplicativo pelas crianças. Esse processo teve como intuito legitimar todas as funcionalidades pensadas para o aplicativo por meio da sua utilização pelo público alvo. Desta forma foram estabelecidas duas formas de avaliação do aplicativo ABC Autismo. A primeira com um questionário direcionado aos pais e profissionais que compõem a associação parceira (AMA-AL) e a segunda voltada para averiguar a utilização da ferramenta por parte das crianças com autismo.

Dentro desta proposta avaliativa foi crucial atestar se, do ponto de vista dos entrevistados, o conteúdo do aplicativo é dinâmico e está em consonância com os preceitos do Programa TEACCH, e também se a interface é intuitiva o suficiente para beneficiar as crianças no processo de utilização do aplicativo. Do ponto de vista da utilização do aplicativo, estávamos interessados em averiguar se as crianças que utilizaram a aplicação reconheciam os princípios da abordagem TEACCH retratados na ferramenta. Para realização desta etapa tivemos o apoio da equipe multidisciplinar da AMA que por possuir mais familiarização com a abordagem e as crianças puderam identificar estas variáveis dentro do processo de validação.

\section{Apresentação do Software}

Muitas das premissas da abordagem manual TEACCH são retratadas no $\mathrm{ABC}$ Autismo, isso se torna evidente por meio de uma interface amigável, elaborada minuciosamente por um design profissional, que redesenhou os elementos comuns utilizados na abordagem manual, desta forma a estruturação das atividades é bastante similar ao modelo manual retratando todo o ambiente utilizado pela criança durante a execução da cada atividade. Tal característica é crucial uma vez que, se tratando de crianças com autismo mudanças drásticas em suas rotinas podem resultar em não aceitação bem como em dificuldades de concentração, o que exige todo um aparato que

\footnotetext{
${ }^{3}$ Scrum é um processo de desenvolvimento iterativo e incremental para gerenciamento de projetos e desenvolvimento ágil de software.

${ }^{4}$ Sprint é a unidade básica de desenvolvimento em Scrum. Sprints tendem a durar entre uma semana e um mês, e são um esforço dentro de uma caixa de tempo de comprimento constante.
} 
os auxiliem nestas questões. Em outras palavras o ambiente precisa ser o mais intuitivo possível, devendo retratar de forma clara quais ações devem ser realizadas pela criança no ato de execução das atividades.

A versão free do aplicativo mobile $\mathrm{ABC}$ Autismo possui 4 níveis de complexidade também conhecidos como níveis de trabalho. Cada nível apresenta 10 atividades sequenciadas em ordem crescente de complexidade. A complexidade dentro da aplicação é medida pela quantidade de estímulos dado a criança, bem como a quantidade e formas de elementos apresentados. Abaixo segue uma breve descrição de cada nível acompanhado de um modelo de tela da aplicação mobile desenvolvida:

- Nível 1 (Figura 1) - É composto de atividades básicas de transposição de figuras que obedecem à ordem estabelecida pela metodologia TEACCH esquerdadireita. Não há critérios para as imagens, contudo as mesmas devem possuir poucos detalhes para evitar a distração do autista. Os elementos utilizados na área de armazenamento, além de simples, contém apenas uma única representação para favorecer o transporte de estímulos. $\mathrm{Na}$ área de resposta há poucos elementos de referência em tamanhos variados de modo a favorecer a resposta correta. Dentro da dinâmica de execução há uma gradual redução da área de resposta válida, que é ativada sempre que a criança obtém sucesso na execução da atividade.

Figura 1 - Tela de atividade do nível 1 no aplicativo ABC Autismo.

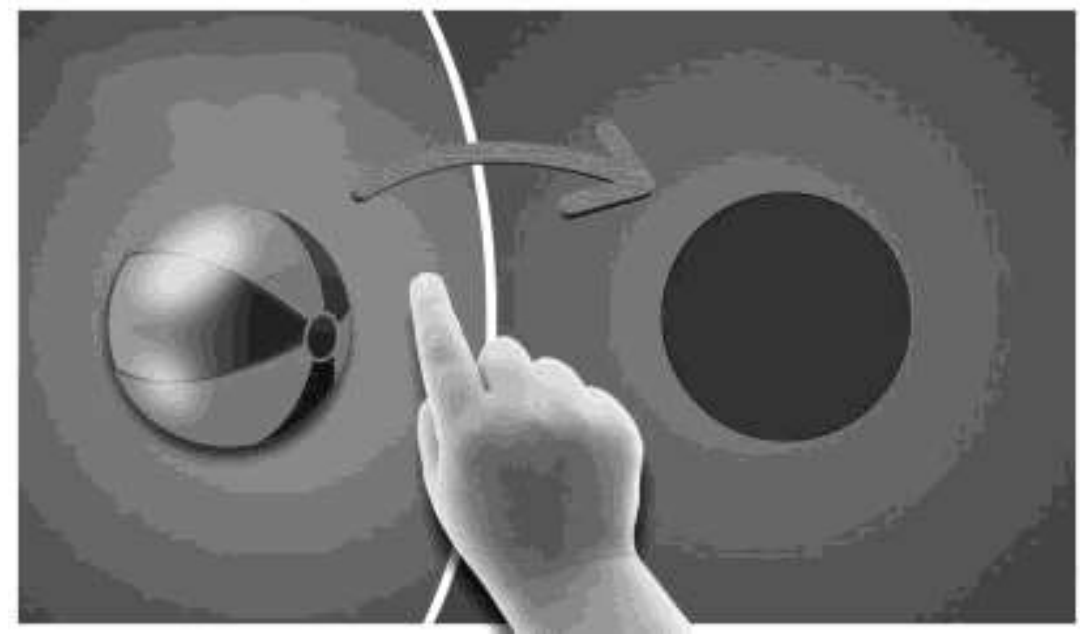

Fonte: Aplicativo ABC Autismo

- Nível 2 (Figura 2) - Exigirá do autista, dentro da aplicação ABC Autismo, o discernimento dos elementos para executar a atividade. $\mathrm{O}$ usuário da aplicação deverá estabelecer critérios para diferenciação de cores, formas e tamanhos dos elementos da área de armazenamento, sendo exigida a devida correspondência destes elementos aos existentes na área de resposta. Neste nível mais estímulos são dados ao autista, uma vez que o mesmo terá que se esforçar cognitivamente para discriminar dos elementos entre si, além de selecionar os mesmos corretamente. Atividades que compõem este nível visam o emparelhamento de imagens, das mais diversas formas e cores. 


\section{CBIE-LACLO 2015}

Anais dos Workshops do IV Congresso Brasileiro de Informática na Educação (CBIE 2015)

\section{Figura 2 - Tela de atividade do nível 2 no aplicativo ABC Autismo.}

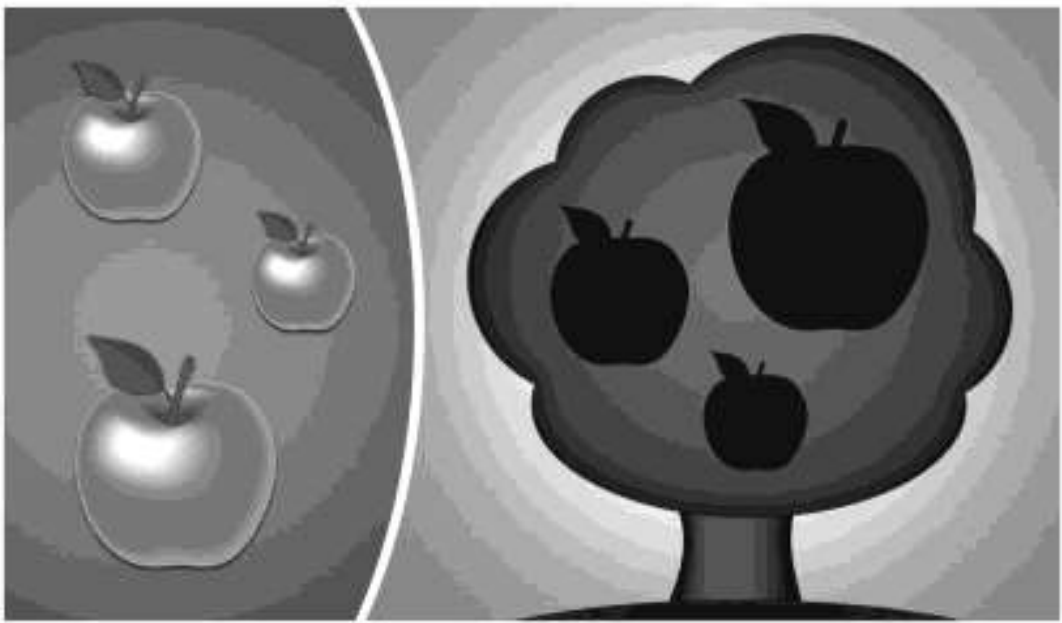

Fonte: Aplicativo ABC Autismo

- Nível 3 (Figura 3) - É voltado para as crianças que já possuem habilidades cognitivas para diferenciação de elementos específicos, exigindo-se agora, a diferenciação de posturas, ações e associações entre som e elemento, imagem e imagem, ação e imagem, letra e letra, número e número, e assim por diante. Atividades de quebra cabeça são comuns neste nível, estas procuram passar para a criança a noção das partes que compõem o todo.

Figura 3 - Tela de atividade do nível 3 no aplicativo ABC Autismo.

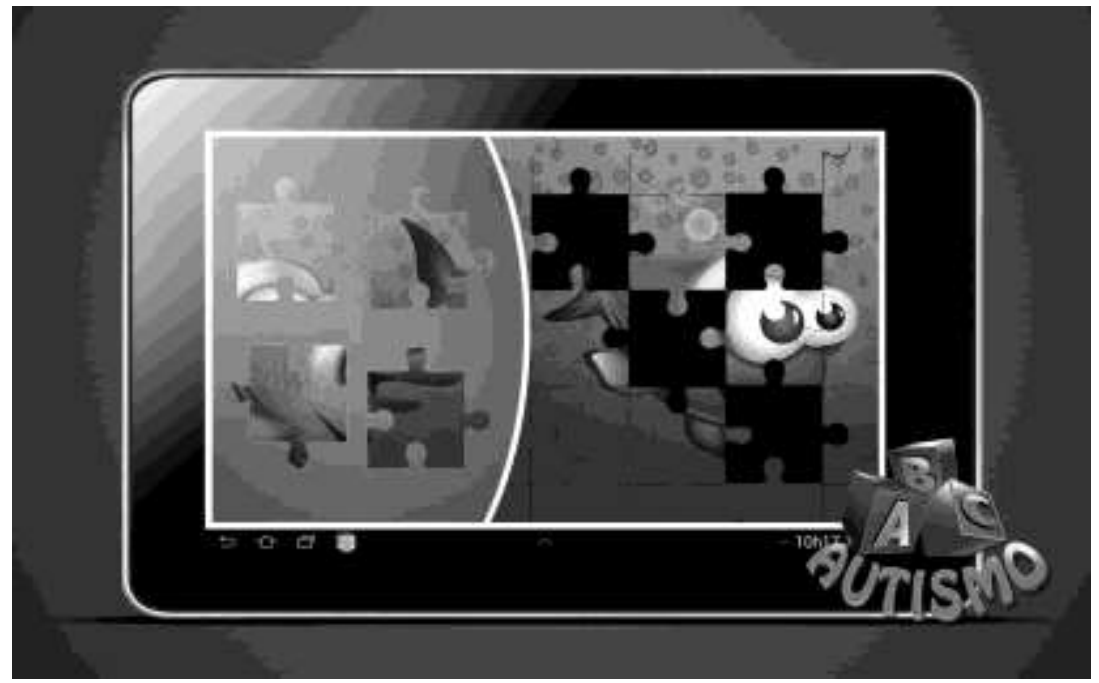

Fonte: Aplicativo ABC Autismo

- Nível 4 (Figura 4) - As atividades que compõem este nível apresentam as noções iniciais de letramento, posicionamento das letras e sílabas nas palavras. É composto de atividades alfabetizadoras, que possuem um nível mais elevado de abstração e simbolismo, visando ensinar ao autista, habilidades básicas de letramento. Assim, atividades de composição de palavras, sequenciamento de números e cruzadinhas são comuns neste nível. 
CBIE-LACLO 2015

Anais dos Workshops do IV Congresso Brasileiro de Informática na Educação (CBIE 2015)

Figura 4 - Tela de atividade do nível 4 no aplicativo ABC Autismo.

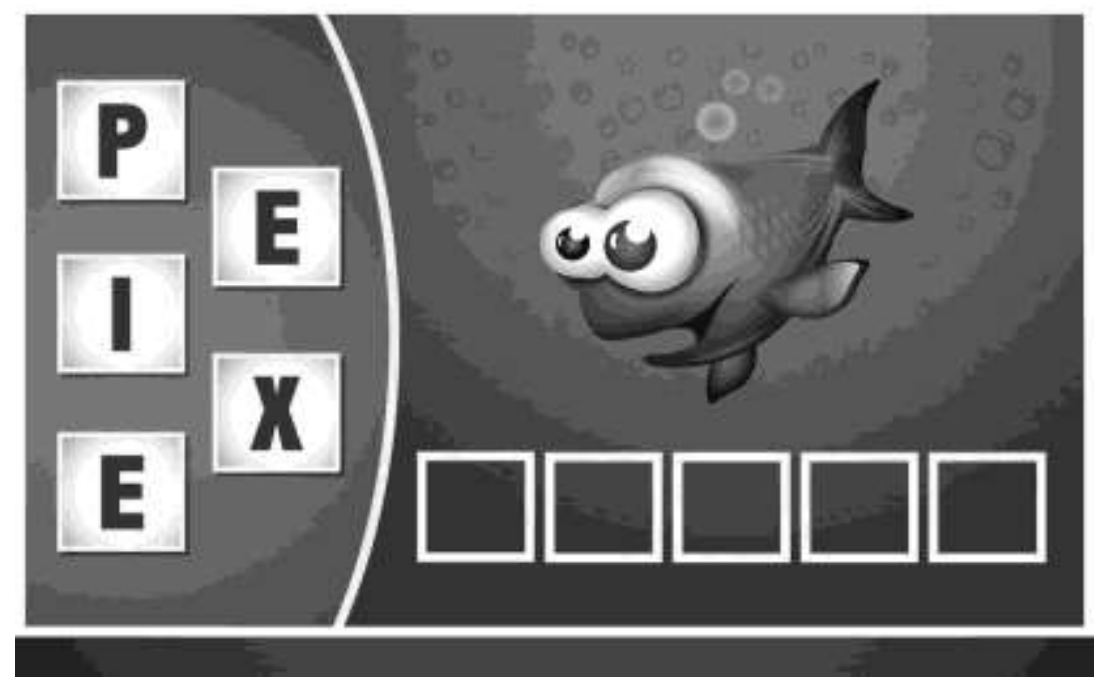

Fonte: Aplicativo ABC Autismo

O aplicativo ABC Autismo encontra-se atualmente na loja de aplicativos Google Play Store, podendo ser acessado pelo link:

https://play.google.com/store/apps/details?id=com.dokye.abcautismo\&hl=pt_BR

A aplicação móvel já possui mais de 10.000 downloads, sendo disponibilizada em inglês, espanhol e português para o público autista em qualquer parte do mundo. Um vídeo demonstrativo do aplicativo pode ser acessado por meio do link: https://www.youtube.com/watch?v=w -3JLz7Y-s.

\section{Considerações Finais}

Muitas vantagens são evidenciadas dentro do cenário de utilização desta aplicação. Inicialmente as características inerentes ao tipo de aplicação desenvolvida, mobile, permitem a transposição da metodologia para os mais variados ambientes, propiciando a ampliação dos benefícios oriundos da utilização da abordagem TEACCH. A criança agora poderá executar a abordagem em tempo integral o que de certa forma potencializará os efeitos interventivos da abordagem.

Além disso, a otimização do tratamento de crianças autistas especialmente no que concerne ao processo de alfabetização seria evidente. Tal melhoria seria fruto da automatização do processo de construção das atividades, que propiciará ao profissional uma otimização de seu tempo, que poderá ser agora gasto com o acompanhamento do quadro evolutivo da criança.

Uma maior adequação das tarefas ao nível de desenvolvimento da cada criança também seria evidente neste cenário, uma vez que a flexibilidade dada ao processo permitiria trabalhar carências especificas da criança através de imagens e estímulos voltados para diminuir gradualmente os déficits apresentados.

A capacidade de personalização das atividades também seria potencializada pela ferramenta, que possibilitará ao profissional, após uma prévia investigação da rotina da criança, utilizar atividades com imagens que representem personagens de maior 
afinidade da criança, o que ajudará a manter a criança atenta a proposta alfabetizadora que está sendo apresentada. A reutilização de elementos em outras tarefas também seria possível por este modelo, uma vez que os mesmos não desgastariam com a manipulação e ação do tempo.

As possibilidades abertas com o uso do aplicativo animaram a todos os envolvidos com o universo autista. Do ponto de vista dos profissionais, como frisado anteriormente, tal aplicação propiciará uma otimização e melhoria de qualidade do serviço oferecido às crianças. Para os pais o aplicativo representa uma nova "esperança" para um efetivo tratamento de seus filhos, pois tal tecnologia é oriunda de um processo sério de pesquisa realizado em parceria com profissionais capacitados com vasta experiência na área, o que de certa forma aumenta a credibilidade e confiabilidade na utilização da ferramenta dentro do processo interventivo. Por fim para os autistas o aplicativo representa um novo brinquedo, iterativo, divertido, onde poderão aprender prazerosamente com imagens e representações comuns ao seu dia a dia.

\section{Referências}

Felicio, V. C. (2007) "O Autismo e o Professor: Um saber que pode Ajudar", Universidade Estadual Paulista Júlio de Mesquisa Filho - Faculdade de Ciências Campus de Bauru. Bauru/SP.

Vila, C; Diogo, S and Sequeira, S. (2009) "Autismo e Síndrome de Asperger", In: Trabalho licenciatura de Psicologia, Instituto Superior Manuel Teixeira Gomes. Portugal.

Farias, E. B and Cunha, M. X. C. (2013) "Protótipo de uma Ferramenta de Software para Apoio no Tratamento de Crianças com Autismo”, In: Simpósio Brasileiro de Sistemas de Informação. Paraíba/Brasil.

ORRÚ, S. E. (2009) “Autismo, linguagem e educação: interação social no cotidiano escolar” Rio de Janeiro: Wak Ed., 2009.

Mello, A. M; Ho, H; Dias, I and Andrade, M. (2013) "Retratos do Autismo no Brasil", Secretaria Nacional de Promoção dos Direitos da Pessoa com Deficiência. $1^{\text {a }}$ Edição.

Farias, E. B; Silva, L. W. C and Cunha, M. X. C. (2014) “ABC Autismo: Um aplicativo móvel para auxiliar na alfabetização de crianças com autismo baseado no Programa TEACCH", Trabalho de Conclusão de Curso, Instituto Federal de Alagoas,. Maceió/Brasil. 